\title{
Quantum Mechanics and the Metrics of General Relativity Paul O'Hara
}

\author{
Dept. of Mathematics, Northeastern Illinois University, 5500 North St. Louis Avenue, \\ Chicago, IL 60625-4699, USA. email: pohara@neiu.edu
}

\begin{abstract}
A one-to-one correspondence is established between linearized space-time metrics of general relativity and the wave equations of quantum mechanics. Also, the key role of boundary conditions in distinguishing quantum mechanics from classical mechanics, will emerge naturally from the procedure. Finally, we will find that the methodology will enable us to introduce not only test charges but also test masses by means of gauges.
\end{abstract}

Pacs: $3.65,4.60$.

\section{Introduction}

There is a fundamental paradigm shift between general relativity and classical mechanics, characterized by the fact that in general relativity the energy-momentum tensor is the effective cause of the space-time curvature, while in classical physics, the structure of space-time is treated as an accidental cause, serving only as a backdrop against which the laws of physics unfold. This split in turn is inherited by quantum mechanics, which is usually developed by changing classical (including special relativity) Hamiltonians into quantum wave equations. In this paper, we will try to remedy this situation by taking the metrics of general relativity as our starting point of quantum mechanics. We will associate wave equations in a natural way with those operators which are duals of differential one-forms rather than with operators derived from a Hamiltonian, thus enabling the structure of space-time itself to determine in a natural and unique way the wave equations of quantum mechanics. Moreover, it is precisely the presence of gauge terms in the form of test masses and charge that permit new laws of physics to emerge independently within the context of the space-time structure in which they are embedded. Throughout the paper, $(\mathcal{M}, g)[4]$ will denote a space-time pair, where " $\mathcal{M}$ is a connected four dimensional Hausdorff manifold" and $g$ is a metric of signature -2 on $\mathcal{M}$. At every point $p \in \mathcal{M}$ 
we can erect a local tetrad $e_{0}(p), e_{2}(p), e_{2}(p), e_{3}(p)$ and a point $x$ will have coordinates $x=\left(x^{0}, x^{1}, x^{2}, x^{3}\right)=x^{a} e_{a}$ in this tetrad coordinate system.[10] We will use Roman letters $a, b, c$ etc. to index coordinates with respect to a tetrad. In this regard, we will refer to $x^{a}$ as the coordinates of $x$. Also for spinors, we can write $\psi=\psi^{i} e_{i}(p)$, where $\psi^{i}$ will represent the coordinates of the spinor with respect to the tetrad at $p$. Also at $p$ we can establish a tangent vector space $T_{p}(\mathcal{M})$, with basis $\left\{\partial_{1}, \partial_{2}, \partial_{3}, \partial_{4}\right\}$ and a dual 1 -form space, denoted by $T_{p}^{*}$ with basis $\left\{d x_{1}, d x_{2}, d x_{3}, d x_{4}\right\}$ at $p$, defined by

$$
d x^{\mu} \partial_{\nu} \equiv \partial_{\nu} x^{\mu}=\delta_{\nu}^{\mu} .
$$

We refer to the basis $\left\{d x^{1}, d x^{2}, d x^{3}, d x^{4}\right\}$ as "the basis of one forms dual to the basis $\left\{\partial_{1}, \partial_{2}, \partial_{3}, \partial_{4}\right\}$ of vectors at $p$. ." [4] Finally, note that Greek letters will be used to represent general coordinate systems and Einsteinian notation will be used for summations.

\section{Metrics and the Dirac equation}

We begin with an intuitive and non-rigorous approach to our methodology by indicating two ways in which quantum mechanical wave equations can be obtained from the metrics of general relativity, without any explicit recourse to Lagrangians or Hamiltonians. We will then combine the results of the two approaches into a mathematical theorem. Later in the next section, we will impose more rigorous constraints, which will enable us to identify the spinor formulation given here with the usual Hilbert Space formulation of quantum mechanics.

1) In a previous paper [9] we have shown that the quantum-mechanical wave equations can be derived as the dual of the Dirac "square-root" of the metric. In other words, if

$$
d s^{2}=g_{\mu \nu} d x^{\mu} d x^{\nu}=\eta_{a b} d x^{a} d x^{b}
$$

where $a$ and $b$ refer to local tetrad coordinates and $\eta$ to a rigid Minkowski metric of signature -2 , then associated with this metric and the vector $\overrightarrow{d s}$ is the scalar $d s$ and a matrix $\tilde{d} s \equiv \gamma_{a} d x^{a}$ respectively, where $\left\{\gamma_{a}, \gamma_{b}\right\}=2 \eta_{a b}$, with $\gamma_{a}$ transforming as a covariant vector under coordinate transformations. Note also that $g_{\mu \nu}(x)=$ $\eta_{a b} e_{\mu}^{a}(x) e_{\nu}^{b}(x)$ with $e_{\mu}^{a}(x)$ forming local tetrads[10] at $x$. Moreover, since $d s$ is an 
invariant scalar, and $\tilde{d s}^{2}=d s^{2}$ we can identify the "eigenvalue" $d s$ with the linear operator $\tilde{d} s$ by forming the spinor eigenvector equation $\tilde{d} s \xi=d s \xi$ (see also Cartan $\mathrm{p}$ 106). This is equivalent to associating the metric

$$
d s^{2}=g_{\mu \nu} d x^{\mu} d x^{\nu}=\eta_{a b} d x^{a} d x^{b}
$$

with the spinor equation:

$$
d s \xi=\gamma_{a} d x^{a} \xi
$$

Note immediately that in agreement with the general theory of eigenvectors, if $\xi$ is a solution so also is $f\left(z_{0}, z_{1}, z_{2}, z_{3}\right) \xi$ where $f$ is any complex scalar valued function. Indeed, there is no reason why $f$ cannot be an $L^{2}$ function, and correspond to a quantum-mechanical wave function.

As previously noted, corresponding to each tangent vector $\frac{\partial}{\partial x^{a}}$, there exists a dual one-form $d x^{a}$. In a similar way, the $\tilde{d} s$ matrix above can be seen as the dual of the expression $\tilde{\partial}_{s} \equiv \gamma^{a} \frac{\partial}{\partial x^{a}}$, where $\gamma^{a}$ is defined by the relationship $\left\{\gamma^{a}, \gamma_{b}\right\}=2 \delta_{b}^{a}$ and the dual map defined by

$$
\left\langle\tilde{d} s, \tilde{\partial}_{s}\right\rangle \equiv \frac{1}{\operatorname{Tr}\left(d x^{i} \partial_{j}\right)} \gamma_{a} \gamma^{b} d x^{a} \frac{\partial}{\partial x^{b}} \equiv \frac{1}{\delta_{i}^{i}} \gamma_{a} \gamma^{b} \frac{\partial x^{a}}{\partial x^{b}}=1
$$

remains invariant. Moreover, if we let $s$ describe the length of a particle's trajectory along a curve $\left(x^{0}(s), x^{1}(s), x^{2}(s), x^{3}(s)\right) \in(\mathcal{M}, g)$ then $s$ can be regarded as an independent parameter with an associated 1-form $d s$, which is the dual of the tangent vector $\partial_{s}$. Note that in terms of the basis vectors for $T_{p}(\mathcal{M})$ and $T_{p}^{*}(\mathcal{M})$ we can write $\partial_{s}=\frac{\partial x^{a}}{\partial s} \partial_{a}$ and $d s=\frac{\partial s}{\partial x^{a}} d x^{a}$. It also follows from this and equation (1) that its dual map is given by $d s . \partial_{s}=\frac{\partial s}{\partial x^{i}} \frac{\partial x^{i}}{\partial s}=1$. Putting these two results together allows us to consider equation (4) as the dual of the equation:

$$
\frac{\partial \psi}{\partial s}=\gamma^{a} \frac{\partial \psi}{\partial x^{a}}
$$

where $\frac{\partial}{\partial s}$ refers to differentiation along a curve parametrized by $s$. We will refer to (6) as a (generalized) Dirac equation and will show later on how it relates to the usual form of this equation. At times, too, we shall refer loosely to it as a "dual wave-equation." 
Now consider the motion of a test particle of mass $m$ along a timelike geodesic. Let $p^{a}=m\left(d x^{a} / d \tau\right)$, where $\tau$ is the proper time (i.e $\left.d s=c d \tau\right)$. Then

$$
d s^{2}=\eta_{a b} d x^{a} d x^{b} \quad \text { is equivalent to } \quad(m c)^{2}=\eta_{a b} p^{a} p^{b} .
$$

This can be expressed in spinor notation by

$$
d s \xi=\gamma_{a} d x^{a} \xi \quad \text { which is equivalent to } \quad \gamma^{a} p_{a} \xi(p)=m c \xi(p) \text {. }
$$

Indeed, if equation (6) is subjected to the constraints of equation (8), as it should be for motion along the timelike geodesic, we find that $\psi=\psi^{i}\left(\int_{x_{0}}^{x(s)} p_{a} d x^{a}\right) e_{i}$ is a solution of equation (6), provided the integration is taken along the curve $s=c \tau$ and $\xi(p)=\frac{d \psi^{i}(p)}{d \tau} e_{i}$ (cf Theorem 1 below). It is also worth noting that if all of $\psi^{i}$ are equal then $\psi=f(x) u$ where $u$ is a spinor independent of $x$, and $f(x)$ is a function. In this particular case the Dirac equation takes on the form

$$
\left(\tilde{\partial}_{s} f\right) u=\frac{\partial f}{\partial s} u
$$

Moreover, in terms of a 4-dimensional (complex) Euclidean space $E^{4}$, this equation can be directly related to the expression[1]

$$
d f=\overrightarrow{d s} . \nabla f=d s \frac{\partial f}{\partial s}
$$

by noting that $\tilde{\partial}_{s} f$ is the matrix form of the vector $\nabla f$. Also, if $\overrightarrow{d s} . \nabla f$ is invariant with respect to both rotations and reflections, then the associated spinor equation can be immediately written in a covariant manner in a natural way. It is sufficient to note that for the Lorentz spinor transformation $D(\Lambda(x))$ applied to the Dirac equation (9), we get

$$
\begin{aligned}
D(\Lambda(x))\left(\tilde{\partial}_{s} f\right) u & =D(\Lambda(x)) \frac{\partial f}{\partial s} u \\
\Rightarrow \quad D(\Lambda(x))\left(\tilde{\partial}_{s} f\right) D^{-1}(\Lambda(x)) D(\Lambda(x)) u & =\frac{\partial f(s))}{\partial s} D(\Lambda(x)) u \\
\Rightarrow \quad \tilde{\partial f_{s^{\prime}}} u^{\prime}\left(x^{\prime}\right) & =\frac{\partial f(x)}{\partial s} u^{\prime}\left(x^{\prime}\right),
\end{aligned}
$$


which expresses the covariance. Note $\tilde{\partial}_{s^{\prime}} \equiv \gamma^{a} \frac{\partial}{\partial x_{a}^{\prime}}$.

2) Another approach to the above formalism is to introduce test particles by means of a gauge term. Specifically, let $(\mathcal{M}, g)$ be a pseudo-Riemannian manifold, with metric tensor $g$ determined by Einstein's field equations. Consider a massless test particle introduced into the field, then by the principle of equivalence, we can choose a local tetrad $\left\{d x_{1}, d x_{2}, d x_{3}, d x_{4}\right\}$, such that the massless test particle travels along a null geodesic given by

$$
0=d x^{a} d x_{a}
$$

which in terms of a spinor basis can be written as

$$
\gamma^{a} d x_{a} \xi=0
$$

Next define the wave equation corresponding to the metric by taking the dual of the 1-form space:

$$
\gamma^{a} \partial_{a} \psi=0
$$

which can be interpreted as the wave equation of a massless particle.

We now introduce a test particle of mass $m$ by means of a minimal principle [10], by adopting the same technique that is usually used to introduce test charges into a field. In other words, let

$$
0=\gamma^{a}\left(\partial_{a}-p_{a}\right) \psi
$$

which in turn gives the fundamental wave equation

$$
\gamma^{a} \partial_{a} \psi=\gamma^{a} p_{a} \psi
$$

This immediately suggests the particular solution

$$
\psi=e^{\int^{x} p^{a} d y_{a}} \xi\left(p_{0}\right) .
$$

Moreover, if the gauge term describes a test particle of mass $m$ moving along a timelike geodesic as defined in (8), then

$$
\gamma^{a} \partial_{a} \psi=m c \psi
$$


Once again, we have obtained a Dirac equation.

To conclude this section, we summarize the results with the following theorem:

Theorem 1 Let $\xi(p)=\left[\frac{d}{d s} \psi^{i}(s)\right] e_{i}$, where mcs $=\int^{x} p^{a} d x_{a}$ along a timelike geodesic then $\gamma^{a} p_{a} \xi=m c \xi$ iff $\psi(p)=\psi^{i}\left(\int^{x} p^{a} d x_{a}\right) e_{i}$ is a solution of

$$
\frac{\partial}{\partial s} \psi(p)=\gamma^{a} \partial_{a} \psi(p)
$$

where $\int^{x} p^{a} d x_{a}$ is Lorentz invariant, and integration is taken along the curve with tangent vector $p^{a}=m \frac{d x^{a}}{d \tau}$, where $\tau$ is proper time.

Proof: Noting that $\psi(p)=\psi^{i}\left(\int^{x} p^{a} d x_{a}\right) e_{i}$ and assuming $\gamma^{a} p_{a} \xi=m c \xi$ then

$$
\begin{aligned}
\gamma^{a} \frac{\partial \psi}{\partial x^{a}} & =\gamma^{a} p_{a} \frac{\partial \psi^{i}}{\partial s} e_{i} \\
& =\gamma^{a} p_{a} \xi \\
& =m c \xi \quad \text { given } \\
& =\frac{\partial \psi}{\partial s}
\end{aligned}
$$

To prove the converse it is sufficient to substitute $\psi(p)=\psi^{i}\left(\int^{x} p^{a} d x_{a}\right) e_{i}$ into $\tilde{\partial}_{s} \psi=$ $\partial_{s} \psi$ to get answer.

Corollary 1 In the case of $\psi^{i}\left(\int^{x} p^{a} d x_{a}\right)=e^{\int^{x} p^{a} d x_{a}}$ then $\gamma^{a} \partial_{a} \psi=m c \psi$.

Proof: Clearly $\frac{\partial \psi}{\partial s}=m c \psi$.

Corollary 2 If $\psi\left(\int^{x} p^{a} d x_{a}\right)=f\left(\int^{x} p^{a} d x_{a}\right) u$ where $u$ is a spinor independent of $x^{a}$ then the equation

$$
\tilde{\partial}_{s} f u=\frac{\partial f}{\partial s} u
$$

has the same solutions as

$$
\tilde{\partial}_{s} \psi=\frac{\partial}{\partial s} \psi
$$


Proof: Substitute.

By way of conclusion, note that if equation (8) is relaxed to incorporate gauge terms other than mass, such as electric charge, then equation (6) can be extended to incorporate the electromagnetic field and in particular, the motion of the electron in a hydrogen atom. We will discuss this in more detail later. For the moment suffice it to say that if $p_{a}=p_{a}^{\prime}-\frac{e}{c} A_{a}$ with $p_{a}^{\prime}$ corresponding to the mass gauge component and subject to the conditions of equation (8) and $A_{a}$ corresponding to the electromagnetic potential, then

$$
\gamma^{a}\left(\partial_{a}+\frac{e}{c} A_{a}\right) \psi=\left(m c+\frac{e}{c} A_{0}\right) \psi
$$

\section{Covariance}

Theorem 1 also enables us to write down a covariant form for the generalized Dirac equation which depends directly upon the covariance of its dual metric equation. We begin by showing that the equation $\tilde{d} s \xi=d s \xi$ is covariant under Lorentz transformations. Specifically, if $d x^{a}=\frac{\partial x^{a}}{\partial x^{\prime b}} d x^{\prime b}=\Lambda_{b}^{a} d x^{\prime b}$ then $\tilde{d} s \xi=d s \xi$ transforms under Lorentz transfomations $D(\Lambda(x))=D(x)$ into

$$
D(x) \tilde{d} s \xi(x)=d s D(x) \xi(x) .
$$

Now the left hand side can be rewritten as

$$
\begin{aligned}
D(x) \tilde{d} \xi \xi & =D(x) \tilde{d} s D^{-1}(x) D(x) \xi(x) \\
& =\tilde{d s^{\prime}} D(x) \xi(x), \quad \text { where } \tilde{d s^{\prime}} \equiv \gamma_{a} d x^{\prime a} \\
& =\tilde{d s}^{\prime} \xi^{\prime}\left(x^{\prime}\right) .
\end{aligned}
$$

Equating the two equations (27) and (30) then gives

$$
\tilde{d s}^{\prime} \xi^{\prime}\left(x^{\prime}\right)=d s \xi^{\prime}\left(x^{\prime}\right),
$$

which establishes the covariance.

To show the covariance of the corresponding Dirac equation, we re-write equation (6) in the form

$$
\left(\tilde{\partial}_{s} \psi^{i}\right) e_{i}=\frac{\partial \psi^{i}}{\partial s} e_{i}
$$


From Theorem 1, we already know that this is equivalent to the the covariant metric $\tilde{d} s \xi=d s \xi$ provided $\xi=\left(d \psi^{i} / d s\right) e_{i}$, where $s$ indicates differention along timelike geodesic parametrized by $s$ (recall $m c s=\int^{x} p_{a} d x^{a}$ ). It follows that $\tilde{d} s \xi=d s \xi$ is covariant iff $\gamma_{a} p^{a} \xi=m c \xi$ is covariant iff $\gamma_{a} p^{a}\left(d \psi^{i} / d s\right) e_{i}=m c\left(d \psi^{i} / d s\right) e_{i}$ is covariant iff equation (32) is covariant with respect to the Lorentz transformation $D(x)$, along the geodesic. Moreover, this latter restriction of motion along a geodesic, may actually be relaxed and the following more general theoren can be proven:

Theorem 2 The Dirac equation defined over the manifold $(\mathcal{M}, g)$ is Lorentz covariant under the transformation $D(x)$ defined with respect to a tetrad $e_{i}(x)$, provided the equation is written in the form

$$
\gamma^{a} \frac{\partial \psi^{i}}{\partial x^{a}} e_{i}(x)=\frac{\partial \psi^{i}}{\partial s} e_{i}(x)
$$

Proof: Let $D(x)$ be a local Lorentz transformation at $x$ then:

$$
\begin{aligned}
D(x) \tilde{\partial}_{s} \psi(s) & =D(x)\left(\tilde{\partial}_{s} \psi^{i}\right) e_{i} \\
& =\left(D(x) \tilde{\partial}_{s} \psi^{i}\right) D^{-1}(x) D(x) e_{i} \\
& =\left(\tilde{\partial}_{s^{\prime}} \psi^{i}\right) e_{i}^{\prime} \\
& =\left(\frac{\partial \psi^{i}}{\partial s}\right) e_{i}^{\prime}
\end{aligned}
$$

Remark: In regular Minkowski space, the covariant form of the generalized Dirac equation can be reduced to the form of equation (6).

\section{Wave Equations for Geodesics}

At this stage the reader may be wondering how the usual formulation of quantum mechanics emerges. Indeed, the wave equations above seem to express the wave equations of classical mechanics more than quantum mechanics, in that there is no expression for Planck's constant $h$, nor does the the expression $i=\sqrt{-1}$ appear with the operators. With regard to the latter point, we note that $i$ could be seen as absorbed into the $\gamma$ matrices, but we postpone a full discussion of this until 
the next section. First, we analyze the solutions of the wave equation for a massless particle from three perspectives to help us better grasp the formal difference between classical and quantum mechanics. Later on, we will formulate the axioms of quantum mechanics as suggested by our analysis.

The linearized metric for a massless particle is given by

$$
0=\gamma^{0} c d t-\gamma^{1} d x_{1}-\gamma^{2} d x_{2}-\gamma^{3} d x_{3}
$$

from which it follows by the canonical correspondence established above that the associated wave equation for the particle is given by:

$$
0=\gamma_{0} \frac{\partial \psi}{c \partial t}-\gamma_{1} \frac{\partial \psi}{\partial x_{1}}-\gamma_{2} \frac{\partial \psi}{\partial x_{2}}-\gamma_{3} \frac{\partial \psi}{\partial x_{3}}
$$

This is the Dirac equation for a massless particle. Squaring this out we get the Klein-Gordan equation for a massless particle :

$$
\frac{1}{c^{2}} \frac{\partial^{2} \psi}{\partial t^{2}}=\sum_{i=1}^{3} \frac{\partial^{2} \psi}{\partial x_{i}^{2}}
$$

Note that, in this formulation, solutions of the massless Dirac equation are also solutions of the usual massless Klein-Gordan equation, although as we shall see later in the potential well problem (section 7), eigenfunction solutions of the Klein-Gordan equation are not necessarily eigenfunctions of the generalized Dirac equation. In this regard, it should also be noted that the Klein-Gordan equation simply prescinds from any discussion of spin or equivalently, it may be considered as the equation for a spin 0 particle. In contrast the Dirac equation has non-zero spin value solutions.

This also raises the question of quantum statistics. It has been noted in a previous paper [8] that Fermi-Dirac statistics is a consequence of indistinguishable particles forming spin-singlet states, while Bose-Einstein statistics follows as a consequence of breaking the rotational invariance associated with the singlet states. Moreover, the easiest way for this breaking to occur is for the spin states of the particles to be statistically independent. It follows as a trivial consequence of the above theory that bosons cannot be second quantized as fermions and fermions cannot be second quantized as bosons, in that particles which are forming spin-singlet states with probability one cannot be considered statistically independent. It also follows that spin 0 particles must obey Bose-Einstein statistics. For example, if $S$ and $T$ 
represent the spin observables of two particles such that $P(S=0)=P(T=0)=1$ then $P(S=0, T=0)=P(S=0) P(T=0)=1.1=1$, and hence the spin observables $S$ and $T$ are statistically independent.

However, it is possible to make and break the spin-singlets. Transposed into the context of quantum field theory this means that the wave function of singlet state particles will have the anti-commutator equal zero while the wave function for statistically dependent particles will have the commutator equal zero. This result clearly differs from the usual form of the spin-statistics theorem, in that the key to understanding the above version of the theorem lies in the rotational invariance of the singlet states, while in the conventional form the theorem associates the different types of statistics with spin values. Nevertheless, in spite of the difference in interpretation and approaches, algebraically the two approaches are easy to reconcile. In Pauli's version of the theorem, the angular momentum operators $L_{i}$, in the case of fermions, obey the Lie Algebra commutator relationships $\left[L_{i}, L_{j}\right]=i \epsilon_{i j k} L_{k}$ and the anti-commutator relationships $\left\{L_{i}, L_{j}\right\}=0$. In the version mentioned above, the spin operators $S$ and $T$ associated with the two distinct particles respectively in a spin-singlet state, are rescaled by defining $S=T=n L$, where $n$ is an integer, and obey the commutator relationships $\left[S_{i}, T_{j}\right]=\left[S_{i}, S_{j}\right]=i n \epsilon_{i j k} S_{k}$. This rescaling allows us to distinguish, for example, spin 1/2 particles from spin 1 particles by taking $n=1$ and $n=2$ respectively in the above relationships. In both cases, the statistics is determined by the commutator relationships, and if in addition, we permit only $n=1$ Pauli's result necessarily follows. However, if we permit different values of $n$ then spin-value ceases to be dependent on commutator relationships, and the generalized Dirac Algebra needs to be interpreted differently.

In this context, rotational invariance offers an alternative interpretation of the underlying Dirac Algebra, both in Pauli's original version of the theorem and the current formulation. For example, in the case of two body "bosonic" composites of spin 0 built out of spin-singlet states, as in the BCS-Anderson theory of superconductivity, the statistics can be explained by partially relaxing the indistinguishability condition. Specifically, n-Cooper pairs can be viewed as obeying the statistics of n-indistinguisable spin-singlets of spin 0 , as distinct from the statistics of $2 \mathrm{n}$-indistinguishable particles forming $\mathrm{n}$ spin-singlets, where in this latter case, particles can switch from one singlet to another. Finally, it is also worth noting that this revised version of the spin-statistics theorem is strongly supported by an argument based on Clebsch-Gordan coefficients. The interested reader is referred to 
the reference above for more details. We now return to our previous discussion.

Since the wave equation emerges from the structure of space-time itself, the question arises as to how to distinguish classical mechanics from quantum mechanics. We investigate this by analyzing the motion of a massless particle in a Minkowski space, subject to different sets of boundary conditions. In the first case we consider the motion of a classical massless particle moving on the $\mathrm{x}$-axis with uniform velocity $c$, but constrained by two mirrors placed at $x=0$ and $x=\xi$ to move uniformly on the interval $[0, \xi]$. We will assume that perfect reflection takes place at the mirrors and that no energy is exchanged. In this case, the equation of motion for a strictly classical particle with position $x=0$ at $t=0$ is given by:

$$
x= \begin{cases}c t-2 n \xi, & \text { for } t \in\left[\frac{2 n \xi}{c}, \frac{(2 n+1) \xi}{c}\right] \\ 2(n+1) \xi-c t & \text { for } t \in\left[\frac{(2 n+1) \xi}{c}, \frac{(2 n+2) \xi}{c}\right],\end{cases}
$$

and its wave function $\psi(x, t)$ takes on the form

$$
\psi(x, t)=\left\{\begin{array}{lll}
\delta[k(x-c t)] & \text { for } \quad x-c t=-2 n \xi \\
\delta[k(x+c t)] & \text { for } \quad x+c t=2(n+1) \xi \\
0 & \text { otherwise. }
\end{array}\right.
$$

The wave function in this case pinpoints the position of the particle with probability 1. Moreover, there is no restriction on the energy (implicit in the term $k$ ) in this case. Theoretically, it may have values ranging from 0 to $\infty$.

However, the classical particle is an idealized situation. In reality, the position of a massless particle constrained to move on the line is unknown and any attempt to know its exact position will be subject to Heisenberg's uncertainty relations, which we will formulate in the next section. In other words, its exact position can not be known in principle, because any attempt to pinpoint it will scuttle the position and defeat the whole purpose of the experiment. The best we can do is to describe the position by means of a uniform probability density $f(x-c t)=1 / \xi$ for $x \in[0, \xi]$ which also suggests writing $\psi(x, t)=e^{ \pm i k(x-c t)} / \sqrt{\xi}$ to preserve both the boundedness and the periodic motion of the particle, as described by the above wave equation. This does not mean that causality is violated nor that the particle does not have an exact position, at least in the above case. It simply affirms that our initial conditions have to be defined statistically, and also in such a way as to reflect the periodic motion of the particle. As a consequence the future evolution of the wave function of the 
system is best interpretated in a statistical way. Finally, note that in this model the energy of the particle can once again vary from 0 to $\infty$ in a continuous manner.

Thirdly, the particle may be constrained to move in a potential well in such a way that the wave function is continuous $(=0)$ at the boundaries. In the case of the above problem, this means that the wave function has harmonic solutions of the form $\psi(\xi, t)=A e^{i \nu^{\prime} t} \sin (k x)$, where $A$ is a constant. Substituting, we will find that $k=\frac{n \pi}{\xi}$ and the photon energy becomes quantized and of the form $E \equiv k c=\nu^{\prime}$. It should be noted that this solution corresponds to the motion of a harmonic oscillator, and is the key to the quantization process associated with quantum field theory in general[7]. Indeed, if we were to rescale our units of energy by defining $\nu^{\prime}=h \nu$, then

$E=h \nu$, with $h$ having units J.s, and the standard wavelength becomes $\lambda=\frac{c h}{E}$. In the next section, $h$ will be introduced in a more formal way.

The purpose of the above three examples is to highlight the importance of the boundary conditions when distinguishing between a classical type problem and a quantum mechanical problem, a point also stressed by Lindsey and Margenau [5]. Classical and quantum laws are not in opposition to each other. There is not one set of laws on the microscopic level and another on the macroscopic. On the contrary, classical and statistical methodologies are complimentary to each other and are in principle, applicable at all levels. However, on the microscopic level, statistical fluctuations will be more pronounced and consequently in practice (and in principle) the effects associated with quantum physics will become more apparent.

\section{Quantum Mechanics and Hilbert Spaces}

The above analysis permits us to better understand something of the difference between quantum mechanics and classical mechanics from the perspective of general relativity. As we have noted, it suggests the difference is to be found in the boundary conditions, which in the case of quantum mechanics is subjected to statistical conditions. With this in mind, we formulate a few axioms which not only respect the manifold structure of general relativity, but also enable us to distinguish quantum mechanics from classical physics, in a formal way.

Essentially what we have noted is that the metric of general relativity forces 
(real) eigenvalue solutions for the free particle of the form

$$
\frac{\partial \psi\left(\int p^{a} d x_{a}\right)}{\partial x_{a}}=p^{a} \psi\left(\int p^{a} d x_{a}\right)
$$

However, since the choice of eigenfunction associated with the specific eigenvalue in this case is not unique, we restrict ourselves for the purpose of quantum mechanics to those eigenfunctions $\psi(t, \mathbf{x})$ such that for each $t, \psi(t, \mathbf{x}) \in L^{2}\left(E^{3}\right) \times H$, where $H$ is a 4-dimensional Hilbert space. Also, we associate the dual of the 1-form $d x$ with the self-adjoint partial differential operator $i \hbar \partial / \partial x$, where $\hbar$ is a constant. Consequently by defining the dual in this way, we not only find that

$$
d x\left(i \hbar \partial_{x}\right) \equiv i \hbar \partial_{x} x=i \hbar
$$

but it can also be linked to the uncertainty principle (see below). At first, this may seem artificial but actually if we look more closely at equation (6) we will find that to associate the operator $-i \partial_{x}$ with a real valued momentum eigenvalue is already implicit it this equation, and indeed is a consequence of the signature of the metric tensor $\eta_{a b}=\left\{\gamma_{a}, \gamma_{b}\right\}$. In particular, if we let $\gamma_{a}=i \alpha_{a}$ for $a=1,2,3$, and set $\gamma_{0}=\alpha_{0}$, then the $\alpha_{a}$ 's are the generators of the Dirac Algebra $S L(2, C)$. Also $\gamma_{a} p^{a}=\alpha_{a}\left(i p^{a}\right)$ and the linearized metric (3) can be written in the explicit form

$$
\tilde{d} s=\alpha_{0} d x^{0}+i \alpha_{1} d x^{1}+i \alpha_{2} d x^{2}+i \alpha_{3} d x^{3}
$$

which in order to maintain the invariant relationship $\left\langle\tilde{d}_{s}, \tilde{\partial}_{s}\right\rangle=1(\operatorname{cf}$ eqn. $(5))$, gives

$$
\tilde{\partial}_{s}=\alpha^{0} \partial_{0}-i \alpha^{1} \partial_{1}-i \alpha^{2} \partial_{2}-i \alpha^{3} \partial_{3}
$$

In other words, if we let $\alpha_{a}$ obey the Dirac Algebra, then we can associate the momentum operator with $-i \partial_{a}$ in a natural way, with eigenvalues $-p_{a}$ for $a=1,2,3$, where $p_{a}=d x^{a} / d \tau$ for each $a$. Finally, let $\hbar=h / 2 \pi$ where $h$ is Plancks constant and rescale the momentum operator by writing $-i \hbar \partial_{a}$ in place of $-i \partial_{a}$ to obtain the usual form of quantum mechanics.

This too may seem artificial, but in reality we are free to choose any scale we wish. This being the case, we choose $\hbar$ because it seems to be the scaling constant, which nature uses. Moreover if we multiply across by $-i \hbar \alpha^{0}$ and note that $\hat{\alpha}_{a} \equiv-i \alpha_{0} \alpha_{a}$ 
obeys the same Dirac Algebra as $\alpha_{a}$, then the Dirac equation (6) can be rewritten as:

$$
-i \hbar \alpha^{0} \frac{\partial \psi}{\partial s}=\left(-i \hbar \partial_{0}-i \hbar \hat{\alpha}^{1} \partial_{1}-i \hbar \hat{\alpha}^{2} \partial_{2}-i \hbar \hat{\alpha}^{3} \partial_{3}\right) \psi
$$

In particular, if we denote the eigenvalue of $i \hbar \partial_{0}$ associated with the eigenfunction $\exp \left(-i \int^{x} p_{a} d x^{a}\right)$ by $p_{0}=-i E /(\hbar c)$ then Cor. 1 gives

$$
\hat{\alpha}^{0} m c^{2} \psi=\left(E-i c \hbar \hat{\alpha}^{1} \partial_{1}-i c \hbar \hat{\alpha}^{2} \partial_{2}-i c \hbar \hat{\alpha}^{3} \partial_{3}\right) \psi
$$

which gives us back the usual form of the Dirac equation. Note too that $\hbar$ has been absorbed into the energy terms.

Finally based on the above discussion, we formulate a few axioms which not only respect the manifold structure of general relativity, but also enable us to distinguish quantum mechanics from classical physics, in a formal way.

Definition 1 Space-time is a four dimensional manifold $(\mathcal{M}, g)$.

Definition 2 At every point $p$ of $\mathcal{M}$ there is a tangent vector space $T_{p}(\mathcal{M})$ with tetrad basis $\left\{-i \hbar \partial_{1},-i \hbar \partial_{2},-i \hbar \partial_{3},-i \hbar \partial_{4}\right\}$ and a dual 1-form space, denoted by $T_{p}^{*}$ with basis $\left\{d x_{1}, d x_{2}, d x_{3}, d x_{4}\right\}$ at $p$, defined by $d x^{a}\left(i \hbar \partial_{b}\right)=i \hbar \frac{\partial x^{a}}{\partial x^{b}}=i \hbar \delta_{b}^{a}$.

Definition 3 Quantum mechanical operators are elements of $S L(2, \mathcal{C})$ Dirac $A l$ gebra, which can be viewed as a representation of the vector spaces $T_{p}$ and $T_{p}^{*}$ on $\mathcal{M}$.

Definition 4 Each element of $S L(2, \mathcal{C})$ algebra acts on the Hilbert Space $L^{2}\left(E^{4}\right) \times$ $H$, where $H$ is a 4-dimensional Hilbert Space. The elements $\psi \in L^{2}\left(E^{4}\right) \times H$ are called the states of the system.

Remark: It follows from the definition of the Hilbert Space that if $\psi \in L^{2}\left(E^{4}\right) \times H$ then for each $t, \psi(t, \mathbf{x}) \equiv \psi_{t}(\mathbf{x})=\psi_{t}^{i} e_{i} \in L^{2}\left(E^{3}\right) \times H$, where each $e_{i} \in H$ and an inner product exists such that $\left\langle\psi_{t}, \psi_{t}\right\rangle=\int\left(\psi^{*}\right)^{i} \psi_{i} d^{3} x$. Moreover, if $\psi_{t}(\mathbf{x})$ is normalized for each $t$ then $\psi^{*} \psi$ can be interpreted as a probability density function for position. 
Lemma 1 Let $d \tilde{f}$ and $\tilde{\partial}_{x}$ be the $S L(2, \mathcal{C})$ representation of $d f \in T^{*}$ and $\partial_{x} \in T$ respectively, then $\left\langle d \tilde{f}, \tilde{\partial}_{x}\right\rangle \psi=\left[\tilde{\partial}_{x}, \tilde{f}\right] \psi$, where $\psi \in L^{2}\left(E^{4}\right)$.

Proof: Note $d \tilde{f}=\frac{\partial f}{\partial x^{a}} d \tilde{x}^{a}$. Therefore

$$
\left\langle d \tilde{f}, \tilde{\partial}_{a}\right\rangle \psi=\frac{\partial f}{\partial x^{a}} \psi=\left[\partial_{a}, f\right] \psi .
$$

The Lemma has been proven.

Lemma 2 (The uncertainty relationships) Let $\tilde{X}=\int_{0}^{x(s)} d \tilde{X}$ and $\tilde{P}=i \hbar \tilde{\partial}_{s}$ be the $S L(2, \mathcal{C})$ representations of position and momentum respectively, defined along a curve of length s. Also let $\bar{X} \equiv \int \psi^{*} \tilde{X} \psi d s, \bar{P} \equiv \int \psi^{*} \tilde{P} \psi d s, \Delta^{2} \tilde{X} \equiv \int \psi^{*}(\tilde{X}-$ $\bar{X})^{2} \psi d s$ and $\Delta^{2} \tilde{P} \equiv \int \psi^{*}(\tilde{P}-\bar{P})^{2} \psi d s$ then $\Delta \tilde{X} \Delta \tilde{P} \geq \frac{\hbar}{2}\left|\int^{s} \psi^{*} \frac{1}{i \hbar}(\tilde{P} \tilde{X}-\tilde{Q} \tilde{X}) \psi d s\right|$. In particular, in the case of the components $\tilde{X}^{a}, \tilde{P}^{a}$ we get $\Delta \tilde{X}^{a} \Delta \tilde{P}^{a} \geq \frac{\hbar}{2}$.

Proof: Usual proof using Cauchy-Schwartz inequality.

\section{Classical Mechanics}

The above formulation lays the ground work for distinguishing classical from quantum mechanics. Indeed, we have seen that quantum theory is highly dependent upon $\hbar>0$ and states $\psi \in L^{2}\left(E^{4}\right)$. Moreover, this suggests that classical mechanics can be obtained by relaxing one of these two conditions either by letting $\hbar \rightarrow 0$ or by choosing $\psi \notin L^{2}\left(E^{4}\right)$ or both. In principle what distinguishes a quantum particle from a classical one is that in contrast to quantum mechanics, the position and momentum of a classical particle can be fully pinpointed and localized. For example, if we reconsider the case of a particle moving uniformly between two mirrors, where the initial position is unknown, then as has already been noted in section 4 , the wave function is given by $\exp (i k x) / \sqrt{\xi}$. However, if this really were a classical situation then in principle both the position and momentum of the particle could be localized and measured exactly, as the distance between the mirrors $\xi$ shrinks to 0 . Moreover, the resulting wave function at any time $t$ would be of the form $\psi(0)=\sum_{k} a_{k} \delta^{(k)}$. This last equation follows from a well known result in distribution theory [2], which states 
Theorem 3 A distribution $T$ which has a support of one point (i.e., is equal to zero except at one point) is a finite linear combination of the Dirac function and its derivatives: $T=\sum_{k} a_{k} \delta^{(k)}$.

Based on this we can formally state that

Definition 5 A classical particle is a particle whose position operator $T$ at any time thas support of one point.

It follows from this definition that the momentum operator $\tilde{P}$ has a support of one point. It also follows from the definition and Theorem 1 that for a classical particle with constant momentum situated at $\left(x_{0}\right)$ on a geodesic, the wave function is given by $\delta^{4}\left(p^{a}\left(x-x_{0}\right)_{a}\right)$. Also the set of operators $T$ in definition 5 , clearly form a subspace of the solution set of the Dirac equation. Indeed, the set $\left\{\delta, \ldots, \delta^{(k)} \ldots\right\}$ is a spanning set for this subspace. Hence, the uncertainty in observing its value is 0 everywhere except at the single point, and the standard deviation $\Delta \tilde{X}$ and $\Delta \tilde{P}$ are also zero. In other words, the uncertainty principle fails for a classical particle. Moreover, in order for nature to circumvent classical solutions, it is sufficient that there be a fundamental unit of wavelength given by $\lambda=\frac{c h}{E}=\frac{2 \pi c \hbar}{E}$, such that $\lambda>0$ whenever $\hbar>0$, which is another way of saying that if $\hbar>0$ then classical solutions need not exist. It also strongly suggests that the process of localizing a particle for measurement is equivalent to confining (at least during the measuring process) the particle to a box. This, in turn, hints that in terms of wave-particle duality, particle properties emerge when we attempt to experimentally localize and isolate the wave, causing a discontinuity in the quantum solutions, closely approximated by delta type functions. We have seen an example of this above, when we considered a particle moving uniformely between two mirrors with wave-function $e^{ \pm i k(x-c t)} / \sqrt{\xi}$.

In concluding this section, we note that classical solutions to the generalized Dirac Equation describing the motion of a particle, can be reduced to distribtions corresponding to point masses as described in Theorem 3 above, and live on a larger space than the $L^{2}$ functions associated with quantum mechanics. To better understand this point, it might be useful to recall the definition of $L^{p}$ spaces, and some of their properties[3]. Consider a fixed measure space $(X, \mathcal{M}, \mu)$. Let $f$ be a measurable function on $X$ such that

$$
\|f\|_{p}=\left(\int|f|^{p} d \mu\right)^{\frac{1}{p}}
$$


then we define

Definition $6 L^{p}(X, \mathcal{M}, \mu)=\left\{f: X \rightarrow \mathcal{C}:\|f\|_{p}<\infty\right\}$.

Also, in general, we can define a bounded linear functional on $L^{p}$ by $\phi_{g}(f)=\int f g$, such that $g \in L^{q}$ where $1 / p+1 / q=1$, and $\phi_{g} \in\left(L^{p}\right)^{*}$, the dual space of $L^{p}$. In the case of $p=2$, the $L^{2}$ space is also a Hilbert space and therefore, its own dual. In turn this allows us to formulate quantum mechanics in a very elegant and simple manner. However, it the case of classical solutions, as described above, the Dirac $\delta$ functional is usually interpreted as a functional on the set of continuous functions of compact support denoted by $C_{c}^{\infty}(X)$, which in turn are dense in $L^{p}$, where $1 \leq p<\infty$. Moreover, in the case of a finite (probability) measure, $\mathrm{E}^{p} \subset \mathrm{E}^{1}$, for $p \geq 1$. With this distinction in mind, it now follows from our formulation that general relativity while pemitting a natural unification of both quantum and classical mechanics by means of the generalized Dirac Equation, also permits a distinction by means of $L^{2}$ functions and distributions which are duals of $L^{1}$ functions.

\section{One dimensional Potential Well}

Consider a quantum particle moving in a Minkowski space such that there is zero potential between $(-a, a)$ and constant potential $V$ for $|x| \geq a$. Then the generalized Dirac equation will in both cases reduce to

$$
\alpha^{0} \hbar \frac{\partial \psi}{\partial c t}-i \hbar \alpha^{1} \frac{\partial \psi}{\partial x}=\hbar \frac{\partial \psi}{\partial s} .
$$

Clearly wave function solutions of the form

$$
\psi= \begin{cases}A e^{\hbar^{-1}[E t-p x]}+B e^{-\hbar^{-1}[E t-p x]} & x \leq-a \\ C e^{i \hbar^{-1}[E t-p x]}+D e^{-i \hbar^{-1}[E t-p x]} & |x|<a \\ F e^{\hbar^{-1}[E t-p x]}+G e^{-\hbar^{-1}[E t-p x]} & x \geq a\end{cases}
$$

can be found, although $\psi$ is not necessarily an eigenfunction of equation (46). Note the $s$ dependency follows from the Lorentz invariant relationship $m c s=E t-p x$, and that in order to satisfy the boundary counditions at $\pm \infty, A=G=0$. Also smooth continuity conditions can be imposed at $\pm a$ to fully solve the system in the usual way. 
On the other hand, if we begin with the conventional form of the Dirac equation as given in Cor 2, namely

$$
\alpha^{0} \hbar \frac{\partial \psi}{\partial c t}-i \hbar \alpha^{1} \frac{\partial \psi}{\partial x}=m c \psi
$$

then solutons of the form $\psi=C e^{i \hbar^{-1}[E t-p x]}+D e^{-i \hbar^{-1}[E t-p x]}$ cannot exist if both $C$ and $D$ are non-zero, as is the case in the conventional potential well problem, where $C= \pm D$. In other words, equation (47) cannot be used to describe a potential well problem. It is also worth noting that if one were to square out either (46) or (47) then both of these equations would have $\psi$ as a permissible solution. This ambiguity arises from the non uniqueness of the square root, especially of the $\alpha^{1}$ matrix which can be defined to be either

$$
\left(\begin{array}{cc}
0 & \sigma_{1} \\
\sigma_{1} & 0
\end{array}\right) \quad \text { or } \quad\left(\begin{array}{cc}
0 & \sigma_{2} \\
\sigma_{2} & 0
\end{array}\right)
$$

where

$$
\sigma_{1}=\left(\begin{array}{cc}
0 & 1 \\
1 & 0
\end{array}\right) \quad \text { and } \quad \sigma_{2}=\left(\begin{array}{cc}
0 & i \\
-i & 0
\end{array}\right)
$$

but not both simultaneously.

\section{Application to the Schwarzschild metric}

We directly apply the above theory to a test particle of mass $m$ moving along a timelike curve parametrized by $\tau$ in a Schwarzschild space, whose metric is defined by [11]:

$$
d s^{2}=B(r) d t^{2}-A(r) d r^{2}-r^{2} d \theta^{2}-r^{2} \sin ^{2} \theta d \phi^{2} .
$$

This allows us to define tetrad coordinates $d x^{0}=B^{1 / 2} d t, d x^{1}=A^{1 / 2} d r, d x^{2}=r d \theta$, $d x^{3}=r \sin (\theta) d \phi$, which on substituting into equation (8) gives

$$
d s \xi=\left[\gamma_{0} B^{\frac{1}{2}} d t-\gamma_{1} A^{\frac{1}{2}} d r-\gamma_{2} r d \theta-\gamma_{3} r \sin (\theta) d \phi\right] \xi .
$$

This is also equivalent to

$$
c \xi=\left[\gamma_{0} B^{\frac{1}{2}} \dot{t}-\gamma_{1} A^{\frac{1}{2}} \dot{r}-\gamma_{2} r \dot{\theta}-\gamma_{3} r \sin (\theta) \dot{\phi}\right] \xi,
$$


which can be seen as the equation of motion along a timelike geodesic. The corresponding wave equation associated with the metric equation (48) is then given by

$$
\frac{\partial \psi}{\partial s}=\gamma^{0} B^{-\frac{1}{2}}(r) \frac{\partial \psi}{\partial t}-\gamma^{1} A^{-\frac{1}{2}}(r) \frac{\partial \psi}{\partial r}-\gamma^{2} \frac{1}{r} \frac{\partial \psi}{\partial \theta}-\gamma^{3} \frac{1}{r \sin \theta} \frac{\partial \psi}{\partial \phi}
$$

To simplify the problem, we assume $\dot{\theta}=\dot{\phi}=0$. Hence, the equation of motion (49) reduces to

$$
\gamma^{0} B^{\frac{1}{2}} \dot{t} \xi-\gamma^{1} A^{\frac{1}{2}} \dot{r} \xi=c \xi
$$

where

$$
\gamma^{0}=\left(\begin{array}{cccc}
1 & 0 & 0 & 0 \\
0 & 1 & 0 & 0 \\
0 & 0 & -1 & 0 \\
0 & 0 & 0 & -1
\end{array}\right) \quad \text { and } \quad \gamma^{1}=\left(\begin{array}{cccc}
0 & 0 & 0 & i \\
0 & 0 & i & 0 \\
0 & i & 0 & 0 \\
i & 0 & 0 & 0
\end{array}\right)
$$

Multiplying out gives

$$
\begin{aligned}
\left(B^{\frac{1}{2}} \dot{t}-c\right) \xi_{0}-i A^{\frac{1}{2}} \dot{r} \xi_{3} & =0 \\
\left(B^{\frac{1}{2}} \dot{t}-c\right) \xi_{1}-i A^{\frac{1}{2}} \dot{r} \xi_{2} & =0 \\
-\left(B^{\frac{1}{2}} \dot{t}+c\right) \xi_{2}-i A^{\frac{1}{2}} \dot{r} \xi_{1} & =0 \\
-\left(B^{\frac{1}{2}} \dot{t}+c\right) \xi_{3}-i A^{\frac{1}{2}} \dot{r} \xi_{0} & =0
\end{aligned}
$$

which can be solved to give

$$
\left(B^{\frac{1}{2}} \dot{t}-c\right) \xi_{0}=i A^{\frac{1}{2}} \dot{r} \xi_{3}
$$

and

$$
\left(B^{\frac{1}{2}} \dot{t}-c\right) \xi_{1}=i A^{\frac{1}{2}} \dot{r} \xi_{2} .
$$

Clearly many solutions are possible. One solution is given by $\xi_{0}=\xi_{1}=i A^{\frac{1}{2}} \dot{r}$ and $\xi_{3}=\xi_{2}=\left(B^{\frac{1}{2}} \dot{t}-c\right)$. Another solution is given by $\xi_{0}=\xi_{1}=\left(B^{\frac{1}{2}} \dot{t}+c\right)$ and $\xi_{3}=\xi_{2}=-i A^{\frac{1}{2}} \dot{r}$. Note that in this latter case, if we substitute into equations (52) and (53) we obtain the equation of motion $B \dot{t}^{2}-A \dot{r}^{2}=c^{2}$. Also, recall from Theorem 1 that $\xi=\frac{\partial \psi^{i}}{\partial s} e_{i}$. Therefore,

$$
\psi^{0}=\psi^{1}=\int\left(B^{\frac{1}{2}} \dot{t}+c\right) d s
$$


and

$$
\psi^{3}=\psi^{2}=-i \int\left(A^{\frac{1}{2}} \dot{r}\right) d s .
$$

In the case of constant motion along a geodesic, the above reduces to $\psi=$ $\psi\left(\int p_{a} d x^{a}\right)=\psi\left(p_{0} x_{0}-p_{1} x_{1}\right)$ where $p_{0}=m B^{\frac{1}{2}} \dot{t}$ and $p_{1}=m A^{\frac{1}{2}} \dot{r}$ are constants of the motion. In particular, in the case of motion inside a potential well of radius $x_{1}(r) \leq l$, we find that $C \exp \left[i\left(p_{0} x_{0}-p_{1} x_{1}\right)\right]+D \exp \left[i\left(p_{0} x_{0}+p_{1} x_{1}\right)\right]$ is a solution of the squared out Dirac equation (expressed in tetrad coordinates)

$$
\hbar^{2} \frac{\partial^{2} \psi}{\partial s^{2}}=\hbar^{2} \frac{\partial^{2} \psi}{\partial x_{0}^{2}}-\hbar^{2} \frac{\partial^{2} \psi}{\partial x_{1}^{2}} .
$$

Denoting the eigenvalues of $i \hbar \frac{\partial}{\partial s}$ by $m c$ and the eigenvalue of $i \hbar \frac{\partial}{\partial x_{0}}$ by $\frac{E}{c}$, this equation reduces to

$$
(m c)^{2} \psi=\left(\frac{E}{c}\right)^{2} \psi+\hbar^{2} \frac{\partial^{2} \psi}{\partial x_{1}^{2}} .
$$

Imposing the boundary conditions $\psi\left(x_{0}, x_{1}(r)=l\right)=0$ gives

$$
\psi=\exp \left(i(E / c) x_{0}\right) \sin \left(p_{1} x_{1}\right)=\exp \left(i(E / c) B^{\frac{1}{2}} t\right) \sin \left(p_{1} x_{1}\right),
$$

with $p_{1} \equiv m k_{n}=\frac{n \pi}{l}$. Therefore the energy levels for the motion of the particle along a geodesic given by $\dot{\theta}=\dot{\phi}=0$ inside the potential well, can be calculated from the equation

$$
(m c)^{2} \psi=\left(\frac{E}{c}\right)^{2} \psi-\left(m k_{n}\right)^{2} \psi
$$

In other words,

$$
E^{2}=m^{2} c^{2}\left(c^{2}+k_{n}^{2}\right)=m^{2} c^{2} B \dot{t}^{2}, \quad n \text { an integer. }
$$

Also, if we permit $l \rightarrow 2 m G / c^{2}$ (the Schwarzschild radius), in such a way that $p_{0}=m\left(1-\frac{2 G m}{c^{2} r}\right)^{\frac{1}{2}} \dot{t}$ and $p_{1}=m\left(1-\frac{2 G m}{c^{2} r}\right)^{-\frac{1}{2}} \dot{r}$ remain constant then we can interpret the above energy levels as the energy levels within a black hole provided we work with spacelike geodesics and not timelike ones.

Finally, to conclude this section, note that in the case of a photon the wave equation along a null geodesic is given by

$$
0=\gamma^{0} B^{-1 / 2}(r) \frac{\partial \psi}{\partial t}-\gamma^{1} A^{-1 / 2}(r) \frac{\partial \psi}{\partial r}-\gamma^{2} \frac{1}{r} \frac{\partial \psi}{\partial \theta}-\gamma^{3} \frac{1}{r \sin \theta} \frac{\partial \psi}{\partial \phi} .
$$




\section{The Hydrogen Atom}

We now apply the techniques of this paper to describe the hydrogen atom. More specifically, we describe the motion of the electron lying within the Reissner-Nordstrom metric [14] of the proton of mass $m_{p}$. Linearizing this metric gives:

$d s=i \alpha_{1}\left(1-\frac{2 G m_{p}}{c^{2} r}+\frac{G e^{2}}{c^{4} r^{2}}\right)^{-\frac{1}{2}} d r+i r\left(\alpha_{2} d \theta+\alpha_{3} \sin \theta d \phi\right)+\alpha_{0}\left(1-\frac{2 G m_{p}}{c^{2} r}+\frac{G e^{2}}{c^{4} r^{2}}\right)^{\frac{1}{2}} c d t$

The corresponding wave equation becomes:

$$
\begin{aligned}
& \frac{\partial}{\partial s} \psi^{\prime}(r, t)=\left\{-i \alpha_{1}\left(1-\frac{2 G m_{p}}{c^{2} r}+\frac{G e^{2}}{c^{4} r^{2}}\right)^{\frac{1}{2}} \frac{\partial}{\partial r}-\frac{i}{r}\left(\alpha_{2} \frac{\partial}{\partial \theta}\right.\right. \\
& \left.\left.+\alpha_{3} \frac{1}{\sin \theta} \frac{\partial}{\partial \phi}\right)+\alpha_{0}\left(1-\frac{2 G m_{p}}{c^{2} r}+\frac{G e^{2}}{c^{4} r^{2}}\right)^{-\frac{1}{2}} \frac{1}{c} \frac{\partial}{\partial t}\right\} \psi^{\prime}(r, t) .
\end{aligned}
$$

We will find on substituting that $\psi^{\prime}=\psi^{\prime}\left(\int^{x} p_{a}^{\prime} d x^{a}\right)$ is a solution, where $p_{a}^{\prime}=p_{a}+\frac{e}{c} A_{a}$ and $A_{a}$ represents the vector potential associated with the electromagnetic field of the proton. In particular, if we seek solutions of the form

$\psi^{\prime}=\exp \left(\frac{i e}{c \hbar} \int A_{a} d x^{a}\right) \psi\left(\frac{i}{\hbar} \int p_{a} d x^{a}\right)$ in tetrad coordinates then this can be reduced to the equation

$$
\left[c \sum_{a=1}^{3} \hat{\alpha}_{a} P_{a}^{\prime}+\alpha_{0}\left(m c^{2}+e V\right)\right] \psi=E \psi,
$$

where $\hat{\alpha}_{a}=-i \alpha_{0} \alpha_{a}, P_{a}^{\prime}=-i \hbar \partial_{a}+\frac{e}{c} A_{a}, m$ is the mass of the electron and $V$ is the electrostatic potential in the rest frame of the proton. Note that this can be written in the usual form of the equation for the hydrogen atom $H \psi=E \psi$, provided $H \equiv\left[c \sum_{a=1}^{3} \hat{\alpha}_{a} P_{a}^{\prime}+\alpha_{0}\left(m c^{2}+e V\right)\right]$. However, there is also an important difference. In this formulation there is the presence of a $\alpha_{0} \mathrm{eV}$, instead of the usual $\mathrm{eV}$, but as we will see below this has advantages.

If we let $\mathcal{G}_{a}=\frac{\partial V}{\partial x^{a}}$, assume $A_{a}=0$ for a $=1,2$ and 3 , then the "square" of this equation, reduces to

$$
\left(-c^{2} \hbar^{2} \sum_{1}^{3} \partial_{a}^{2}+\left(m c^{2}+e V\right)^{2}+i \hbar e c \hat{\alpha}_{a} \alpha_{0} \mathcal{G}_{a}\right) \psi=E^{2} \psi
$$


Noting that $-i \hbar \hat{\alpha_{a}} \alpha_{0}=\alpha_{a}$ which can also be seen as generators of the Dirac Algebra, this can be rewritten as:

$$
\left(-c^{2} \hbar^{2} \sum_{1}^{3} \partial_{a}^{2}+\left(m c^{2}+e V\right)^{2}-\hbar e c \alpha_{a} \mathcal{G}_{a}\right) \psi=E^{2} \psi
$$

Contrast this with conventional relativistic quantum mechanics where we would have found

$$
\left(-c^{2} \hbar^{2} \sum_{1}^{3} \partial_{a}^{2}+m^{2} c^{4}-i \hbar e c \alpha_{a} \mathcal{G}_{a}\right) \psi=(E+e V)^{2} \psi .
$$

Notice that the complex number coefficient associated with the electric moment $\mathcal{G}$ in this latter equation has been replaced with a real coefficient in equation (66). In other words, the spin electric moment $\frac{\hbar e}{2 i m c}$, which is a complex number, can be replaced with a real spin term $\frac{\hbar}{2 m c}$ thus removing the ambiguity normally associated with electron spin in the hydrogen atom. For example, Lindsey and Margenau [6] warn us "not to take the electron spin to literally", because of the presence of the imaginery term. Happily, we can say that with the above approach the difficulty is resolved.

\section{Conclusion}

In this paper we have attempted to unify general relativity and quantum mechanics by viewing any metric as a dual of a wave equation. We have noted that the resulting wave equation contains the usual Dirac equation of quantum mechanics as a special case. We have also noted that the difference between quantum and classical mechanics seems to lie in boundary conditions, with quantization (as distinct from quantum theory) emerging when the wave function is confined to a finite domain with continuous boundary conditions, and classical mechanics being the result of delta-function type solutions for the wave equation. Indeed, this particular approach also highlights the harmonic oscillator as a natural starting model for quantum field theory, by confining the quantum particle to a box. Of course, our use of the word "confined" differs from the usual non-Abelian gauge theory usage. Here the word, 
confined means considering solutions of the generalized Dirac equation constrained by periodic boundary conditions. Nevertheless it would not surprise me if in the future the two different types of confinement end up being linked.

Overall, our approach was able to duplicate the standard results of quantum mechanics but in addition, we were able to remove the anomaly of an imaginery electric moment, when solving the hydrogen atom problem. This result in itself, should be sufficient to encourage further development. I would also hope that further studies will be carried out on the relationship between confinement problems in general and quantum boundary conditions.

\section{References}

[1] Élie Cartan, The Theory Of Spinors, Dover, 134(1966).

[2] Duff and Naylor, Differential Equations Of Applied Mathematics, Wiley \& Sons, 71(1966).

[3] Gerald Folland, Real Analysis, Wiley \& Sons, 170-210(1884).

[4] Hawking \& Ellis, The large scale structure of space-time, Cambridge University Press, 17(1995).

[5] Robert Lindsay and Henry Margenau, Foundations of Physics, Dover, 46$55(1957)$.

[6] Robert Lindsay and Henry Margenau, Foundations of Physics, Dover, $511(1957)$.

[7] Peter W. Milonni, The Quantum Vacuum, Academic Press, 331-354(1994).

[8] Paul O'Hara, Rotational invariance and the Spin-Statistics Theorem, Foundations of Physics, 1349-1368(2003).

[9] Paul O'Hara, Wave-particle duality in general relativity, Nuovo Cimento, 799810(1996).

[10] Lochlainn O'Raifeartaigh, The Dawning of Gauge Theory, Princeton University Press, 112(1997). 
[11] Steven Weinberger, Gravitation and Cosmology: Principles and Applications of the General Theory of Relativity, Wiley \& Sons,179(1972). 